\title{
Can we use the neutrophil-to-lymphocyte ratio, platelet-to-lymphocyte ratio, and mean platelet volume values for the diagnosis of anterior uveitis in patients with Behcet's disease?
}

\author{
This article was published in the following Dove Press journal: \\ Therapeutics and Clinical Risk Management \\ 17 July 2017 \\ Number of times this article has been viewed
}

\begin{abstract}
Atil Avci'
Deniz Avci

Fatma Erden ${ }^{3}$

Ertas Ragip'

Ali Cetinkaya ${ }^{2}$

Kemal Ozyurt ${ }^{1}$

Mustafa Atasoy'

'Department of Dermatology and Venereology, Kayseri Training and Research Hospital, Kayseri, Turkey; ${ }^{2}$ Internal Medicine Department, Kayseri Training and Research Hospital, Kayseri, Turkey; ${ }^{3}$ Department of Dermatology and Venereology, Cubuk State Hospital, Ankara, Turkey
\end{abstract}

Introduction: The purpose of this study was to compare the value of hematological parameters, neutrophil-to-lymphocyte ratio (NLR), platelet-to-lymphocyte ratio (PLR), and mean platelet volume (MPV), as indicators of anterior uveal segment involvement in patients with Behcet's disease (BD).

Patients and methods: Hospital-based records of a total of 912 patients with BD from the dermatology clinic and healthy volunteers from the checkup clinic were assessed retrospectively. After applying the exclusion criteria of the study, 71 of the BD patients with anterior uveitis, 69 of the BD patients without ophthalmological pathology and 151 healthy volunteers were included in the study. MPV, PLR, and NLR values of patients and healthy volunteers were compared.

Results: All MPV, PLR, and NLR values of patients who had anterior uveitis were significantly higher than those of other patients and healthy volunteers. Statistically, considering area under curves (ratio): NLR was 0.725 (0.653-0.797), $P<0.001$; PLR was 0.600 (0.523-0.676), $P=0.012$, and MPV was 0.358 (0.279-0.437), $P<0.001$.

Conclusion: MPV, PLR, and NLR are all valuable for assessment of anterior uveal segment involvement in patients with BD. However, the NLR seems to be better than the PLR and MPV for indicating anterior uveitis due to BD.

Keywords: Behcet's disease, NLR, PLR, MPV, uveitis

\section{Introduction}

Behcet's disease (BD) is a multi-systemic disease characterized by oral and genital aphthous ulcers and skin lesions, and it can also involve many organs and systems, such as the eyes and central nervous system. ${ }^{1}$ BD was first described by Hulusi Behcet in 1937 , but the etiopathogenesis of the disease has not yet been fully elucidated. ${ }^{2}$ Until 2014, many researchers adopted the criteria of the International Study Group, established in 1990, for the diagnosis of BD. ${ }^{3}$

Bilateral, recurrent, non-granulomatous uveitis is a well-known ocular manifestation of BD. Retinal vasculitis and involvement of the posterior segment (eg, retinal hemorrhage, optic atrophy, hypopyon, and choroiditis) may also be seen in BD. ${ }^{4}$

There is no specific laboratory test for diagnosing BD. Many studies have been conducted on tests which may reflect disease activation, be helpful in monitoring treatment efficiency, or predict potential complications of BD. ${ }^{5}$ In the literature, a limited number of studies have attempted to identify an inflammatory marker which
Correspondence: Fatma Erden Cubuk State Hospital, Department of Dermatology and Venereology, Cumhuriyet Mh., Ankara Bulvarı No 54, Ankara 06760, Turkey Tel +905327803805

Email doktor_ayyildiz@hotmail.com 
can predict uveitis. Türkçü et al reported that initially, mean platelet volume (MPV) was significantly lower in patients with active uveitis than in controls, but the significance disappeared over time. ${ }^{6,7}$

In general, BD progresses with inflammation, which has been emphasized in the pathogenesis of uveitis. This raises the question of whether inflammation markers can be helpful in identifying patients with uveitis. The platelet-to-lymphocyte ratio (PLR) has been suggested as a predictor of several conditions, especially cancer and the inflammatory conditions discussed in the following sections. ${ }^{8-13}$ The neutrophil-tolymphocyte ratio (NLR) has been evaluated in some studies on BD. ${ }^{14}$ This issue was also researched by our group while studying anterior uveitis related to $\mathrm{BD}(\mathrm{BD}+\mathrm{AU}){ }^{15}$

In this study, we investigated the MPV, NLR, and PLR in order to determine which one is best for indicating anterior uveitis in patients with BD.

\section{Patients and methods}

Data were retrospectively gathered from 912 patients presenting to the Kayseri Research and Training Hospital and diagnosed with $\mathrm{BD}$ using international diagnostic criteria. Of 912 patients, 217 with anterior uveitis but without ocular signs were considered eligible, as they fulfilled the International Working Group's criteria for ophthalmological examination.

Exclusion criteria for patients were: if ophthalmologic examination of patients was not performed, eye disease other than anterior uveitis was present, patients with cardiovascular involvement, renal failure, cardiac failure, vascular thrombosis, a history of anticoagulant use, or concurrent infections, due to the potential effects of these conditions on the MPV, PLR, or NLR. These patients were also excluded if active BD findings (arthritis, gastrointestinal system involvement, etc.) were noted, which would cause inflammatory responses in the patient and could alter hematologic parameters. We attempted to exclude patients who were noted to be in the inflammatory process, either independently of the BD or depending on the BD activity. Another exclusion criterion was the timing of blood testing; patients whose blood samples were not collected at the same time as the ophthalmological examination were not included in the study.

Thus, 71 patients with a diagnosis of anterior uveitis $(\mathrm{BD}+\mathrm{AU})$ and $69 \mathrm{BD}$ subjects with normal ocular findings (BD) at the time of the blood tests were included in the $\mathrm{BD}+\mathrm{AU}$ and $\mathrm{BD}$ groups, respectively. A third group, the healthy controls, consisted of 151 patients. We carefully screened healthy volunteer patients for chronic liver disease, chronic kidney disease, infectious disease, or chronic illness which could cause inflammation. The group consisting of patients with BD without any ophthalmologic findings was noted as BD. The group consisting of BD patients who had anterior uveitis was classified as BD + AU. Patients were excluded from the study if their tests were not performed simultaneously with anterior uveitis diagnosis. The PLR was determined by dividing the platelet count by the lymphocyte count. The NLR was determined by dividing the neutrophil count by the lymphocyte count.

This study was approved by the Kayseri Training and Research Hospital Ethics Committee. The ethics committee obtained the necessary approvals for the use of medical records of patients and volunteers. Patient consent to review their medical records was not required by the Kayseri Training and Research Hospital Ethics Committee, as all data were de-identified and anonymized.

\section{Statistical analysis}

The continuous data were analyzed to find the mean, standard deviation, median, and percentages. Student's $t$-test was used to compare continuous variables among the studied groups. The Mann-Whitney $U$ test was used to compare the medians of numerical variables with a skewed distribution. One-way ANOVA and Kruskal-Wallis tests were used to compare the parameters in all three groups according to their distribution. A Bonferroni correction was performed. A chi-square test was used to compare categorical variables. Pearson correlation analysis was used to determine the relationships between the patient and control groups. ROC curves were used to evaluate the performance of variables in indicating the presence of anterior uveitis in $\mathrm{BD}$ patients. A $P$-value of $<0.05$ was considered to be significant. All statistical analyses were performed using Statistical Package Program for Social Sciences, version 23.0 (IBM Corporation, Armonk, NY, USA).

\section{Results}

The mean age was $40.41 \pm 14.20$ years in the healthy group, $39.44 \pm 10.28$ years in the BD group, and $37.44 \pm 10.82$ years in the BD + AU group $(P=0.244)$. Females comprised $23.2 \%$ of the BD group, and $76.8 \%$ of the patients in the BD + AU group were male $(P<0.001)$. The distribution of the healthy subjects according to sex was $75.6 \%$ male $/ 24.4 \%$ female. Features of the control and patient groups are summarized in Table 1.

\section{PLR feature}

Values of the PLR were significantly different among groups $(P=0.040)$; the mean PLR value was $124.45 \pm 33.12$ 
Table I Comparison between Behcet's disease groups with and without anterior uveitis

\begin{tabular}{|c|c|c|c|c|}
\hline \multirow[t]{2}{*}{ Parameters } & \multirow[t]{2}{*}{ Healthy controls $(n=\mid 5 I)$} & \multicolumn{2}{|l|}{ Behcet's disease (BD) groups } & \multirow[t]{2}{*}{$P$-value } \\
\hline & & BD without uveitis $(n=69)$ & BD with uveitis $(n=7 I)$ & \\
\hline Age (years)* & $40.4 I \pm 14.20$ & $39.28 \pm 14.20$ & $37.44 \pm 10.82$ & $P=0.244$ \\
\hline \multirow[t]{2}{*}{ WBC $(I / \mu L)^{\#}$} & $7,200(6,140-8,610)^{e}$ & $6,747(6,045-7,765)^{f}$ & $7,800(6,220-9,080)^{\mathrm{e}, \mathrm{f}}$ & $P=0.042^{e}$ \\
\hline & & & & $P=0.002^{f}$ \\
\hline \multirow[t]{3}{*}{ Neutrophil $(I / \mu \mathrm{L})^{\#}$} & $4,180(3,520-5,100)^{g, h}$ & $3,800(3,285-4,430)^{g . i}$ & $4,600(3,800-6,000)^{h, i}$ & $P=0.003^{g}$ \\
\hline & & & & $P=0.009^{h}$ \\
\hline & & & & $P<0.00 I^{i}$ \\
\hline \multirow[t]{2}{*}{ Lymphocyte $(\mathrm{I} / \mu \mathrm{L})^{\#}$} & $2,270(1,920-2,680)^{i}$ & $2,340(1,950-2,785)^{k}$ & $2,100(1,720-2,500)^{\mathrm{j}, \mathrm{k}}$ & $P=0.018^{j}$ \\
\hline & & & & $P=0.009^{k}$ \\
\hline Platelets $(\mathrm{I} / \mu \mathrm{L})^{\#}$ & $281,000(243,000-317,000)$ & $277,000(227,000-335,000)$ & $273,000(239,000-298,000)$ & $P=0.857$ \\
\hline \multirow[t]{2}{*}{$\mathrm{MPV}(\mathrm{fL})^{\#}$} & $9.8 I \pm\left. I .1\right|^{\mathrm{cm}}$ & $9.35 \pm 1.36^{\mathrm{m}}$ & $8.97 \pm I .4 I^{c}$ & $P<0.00 I^{c}$ \\
\hline & & & & $P=0.032^{\mathrm{m}}$ \\
\hline $\mathrm{PLR}^{*}$ & $124.45 \pm 33.12^{\mathrm{b}}$ & $125.15 \pm 4 \mid .44$ & $137.37 \pm 38.16^{b}$ & $P=0.040^{b}$ \\
\hline $\mathrm{NLR}^{\#}$ & $1.88(1.52-2.26)^{d}$ & $1.54(1.38-1.8 \mathrm{I})^{\mathrm{d}}$ & $2.37(0.96-6.36)^{d}$ & $P<0.00 I^{d}$ \\
\hline Sex M/F (\%) & $56.3 / 43.7$ & $23.2 / 76.8^{\mathrm{a}}$ & $66.2 / 33.8^{\mathrm{a}}$ & $P<0.000 I^{a}$ \\
\hline
\end{tabular}

Notes: *Mean \pm SD, ${ }^{*}$ median $(25-75$ percentiles). Statistically significant values are presented in bold. Superscript letters define the significant $P$-values of pairwise comparisons.

Abbreviations: WBC, white blood cells; MPV, mean platelet volume; PLR, platelet-to-lymphocyte ratio; NLR, neutrophil-to-lymphocyte ratio; BD, Behcet's disease; $\mathrm{M}$, male; F, female.

in the healthy controls, $125.15 \pm 41.44$ in the BD group, and $137.37 \pm 38.16$ in the $\mathrm{BD}+\mathrm{AU}$ group. With a Bonferroni correction applied, the difference between the healthy controls and the BD group was not statistically significant $(P=1.000)$, the difference between the healthy controls and the $\mathrm{BD}+$ AU group was statistically significant $(P=0.043)$, and the difference between the $\mathrm{BD}$ and $\mathrm{BD}+\mathrm{AU}$ groups was not statistically significant $(P=0.145)$. The AUC (ratio) value of the PLR was $0.600(0.523-0.676)$, with $P=0.012$.

\section{MPV feature}

The mean MPV was $9.81 \pm 1.11 \mathrm{fl}$ in the healthy control group, $9.35 \pm 1.36 \mathrm{fl}$ in the BD group, and $8.97 \pm 1.41 \mathrm{fl}$ in the BD + AU groups; the groups were statistically significantly different $(P<0.001)$. With a Bonferroni correction applied, the difference between the healthy controls and the BD group was statistically significant $(P=0.032)$, the difference between the healthy controls and the $\mathrm{BD}+\mathrm{AU}$ group was statistically significant $(P<0.001)$, and the difference between the $\mathrm{BD}$ and $\mathrm{BD}+\mathrm{AU}$ groups was not statistically significant $(P=0.235)$. The AUC value of the MPV was $0.358(0.279-0.437)$, with $P<0.001$.

\section{NLR feature}

When the NLR values of the groups were compared, the median value was 2.37 (0.96-6.36) in the $\mathrm{BD}+\mathrm{AU}$ group and $1.54(1.38-1.81)$ in the BD group. The median NLR of the healthy controls was $1.88(1.52-2.26)$. The groups were significantly different $(P<0.001)$. In pair-wise comparisons, the difference between the healthy controls and the BD group was statistically significant $(P<0.001)$, the healthy controls and the $\mathrm{BD}+\mathrm{AU}$ group were significantly different $(P<0.001)$, and the $\mathrm{BD}$ and $\mathrm{BD}+\mathrm{AU}$ groups were significantly different $(P<0.001)$. The AUC value of the NLR was $0.725(0.653-0.797)$, with $P<0.001$. The NLR of 2.04 had $67.6 \%$ sensitivity and $69.1 \%$ specificity for anterior uveitis.

ROC analyses were performed to compare the ability of the MPV, PLR, and NLR to indicate anterior uveitis and are summarized in Figure 1.

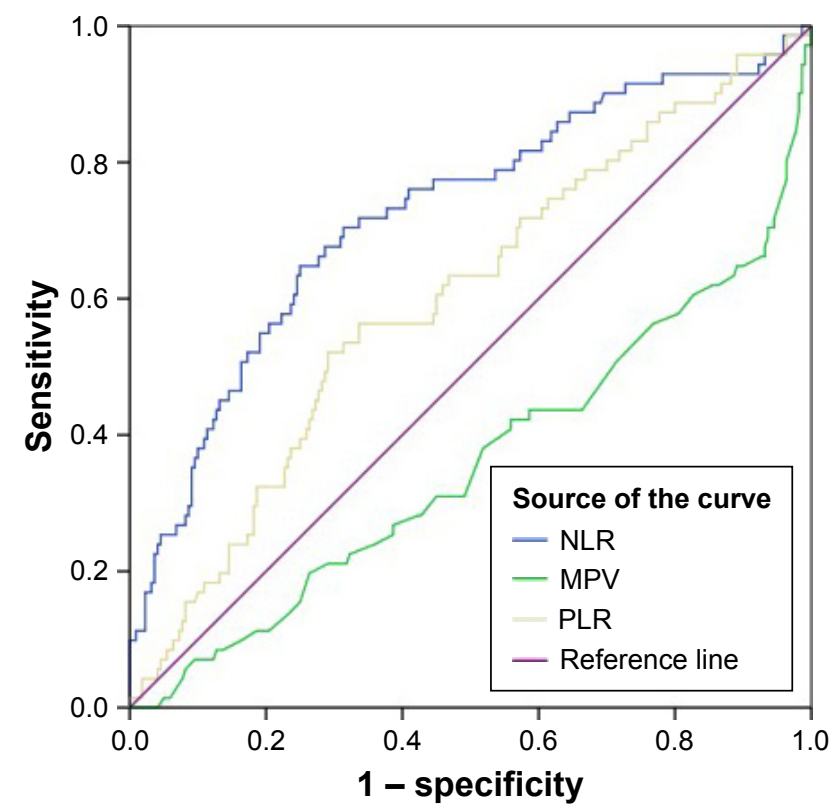

Figure I Receiver operating characteristic curves for neutrophil to NLR, MPV and PLR.

Note: Diagonal segments are produced by ties.

Abbreviations: MPV, mean platelet volume; PLR, platelet-to-lymphocyte ratio; NLR, neutrophil-to-lymphocyte ratio. 


\section{Discussion}

In this study, we investigated changes in some hematological parameters which point to inflammation in the context of anterior uveitis, a complication of BD. Male patients represented the majority of the BD + AU group, consistent with previous reports. ${ }^{16}$ The age of the patients in each of the three groups was similar.

In the pathogenesis of $\mathrm{BD}$, neutrophils are believed to play an important role. It is thought that neutrophil hyperactivation, which can be confirmed by the up-regulation of CD10, CD11a, and CD14 on cell surfaces, plays an important role in the pathogenesis of BD by increasing chemotaxis, phagocytosis, superoxide generation, and myeloperoxidase levels. ${ }^{17-21}$ In BD, pro-inflammatory cytokines secreted by T cells or APCs, such as TNF- $\alpha$, IL- 8 , IFN- $\gamma$, or G-CSF, seem to be responsible for the priming of neutrophils, but the underlying mechanism is not yet clear. ${ }^{22,23}$ Current data suggest that platelets interact with endothelial cells and leukocytes, leading to release of inflammatory substances which cause monocyte adhesion and transmigration. ${ }^{24,25}$

The NLR, PLR, and MPV have been investigated to find out which of them is most valuable in the diagnosis of BD + AU. We did not find any other studies which had investigated these values for anterior uveitis before we completed our study and submitted it for publication. However, there have been recent closed-design studies examining BD and ocular complications. For the sake of clarity, we will discuss them under separate headings for each parameter.

\section{MPV}

The MPV is a direct indicator of platelet function and exhibits an inverse correlation with platelet count. ${ }^{26}$ As stated in the introduction, changes in the MPV in many different conditions have been researched. While the MPV was not found to be associated with disease and/or disease activity in some of these conditions, it is increased or decreased in others. An increased MPV has been associated with increased thrombotic disposition. ${ }^{27}$ Also, a reduced MPV has been found in patients with rheumatoid arthritis, ankylosing spondylitis, and systemic lupus erythematosus arthritis when compared to controls. ${ }^{28,29}$ A higher MPV in patients with active BD has also been reported. ${ }^{30}$ The role of the MPV in differentiation between $\mathrm{BD}$ and recurrent oral aphthous ulcers was also investigated, but no evidence for the effectiveness of the MPV in such differentiation could be obtained. ${ }^{23}$ In a recent study, Türkçü et al examined the MPV in 36 BD patients with ocular findings and found no significant change. ${ }^{6}$ However, in a very recent study from Turkey, MPV and severity of BD were found to be correlated with each other. ${ }^{31}$ In that study, complications were evaluated as a whole (eg, arthritis and complications of the eyes, large vessels, and central nervous system).

It is important to note that the current study focuses only on anterior uveitis, and the sample size is larger than previous studies. In both the $\mathrm{BD}$ and $\mathrm{BD}+\mathrm{AU}$ groups, the MPV values were found to be low even though they were not statistically different from the healthy group. However, the difference between the median MPV in the BD and BD + AU groups was not statistically significant. As was concluded in a previous study, we found no relationship between the MPV and anterior uveitis. ${ }^{6}$

\section{PLR}

In recent years, the PLR has emerged as a marker which can be readily used for several diseases. Studies indicate a significant increase in the PLR in cardiovascular and oncologic diseases. In a recent study, it was shown that the PLR is a prognostic factor in non-ST-elevation myocardial infarction. ${ }^{32}$ A number of studies, including the study conducted by Smith et al, have shown that the PLR is a prognostic factor in periampullary pancreatic carcinoma. ${ }^{8}$ Asher et al reported that the PLR is a novel marker which may predict ovarian cancer. ${ }^{9}$ In a recent study, the PLR was found to be significantly higher in dipper than in nondipper hypertension. ${ }^{10}$ A study by Gary et al concluded that an increased PLR is associated with critical ischemia of the extremities in patients with peripheral arterial occlusive disease. ${ }^{11}$ Recently, a study from Turkey reported that the PLR is associated with the coronary slow flow phenomenon. ${ }^{12}$ We found that the PLR value was significantly higher in the $\mathrm{BD}+\mathrm{AU}$ group than in the healthy group. There was no significant difference between the $\mathrm{BD}$ and $\mathrm{BD}+\mathrm{AU}$ groups or between the $\mathrm{BD}$ and healthy control groups.

\section{NLR}

The NLR is accepted as an indicator of worsening or recurrence of chronic diseases. In the literature, an increased NLR is mostly used as a negative prognostic factor in cardiovascular diseases and cancers. ${ }^{33-35}$ It has also been investigated in respiratory system diseases, pulmonary tuberculosis, and serious infections and found to be higher in all of them. ${ }^{36-38}$ Similarly, systemic inflammation has been reported as a significant factor in metabolic syndromes, including obesity and diabetes mellitus. ${ }^{39,40}$ Cananzi et al reviewed recent evidence that immunological phenomena may explain the unexpected positive response rate in patients with advanced disease. A simple estimation of the NLR has been advocated 
as a prognostic marker for several cancers; eg, it is useful in metastatic melanoma. ${ }^{41}$ In the recent study by our group, we showed that the NLR changes significantly in patients with anterior uveitis. ${ }^{15}$ Similarly in a recent study, Balkarli et al concluded that NLR may be a simple, inexpensive, and convenient diagnostic marker of active BD. ${ }^{42}$ And recently, Yüksel et al concluded that NLR levels were higher in patients with active ocular BD compared to the inactive ocular BD group. ${ }^{43}$

As a limitation, since it was a retrospective study, it was not possible to report clearly the treatments applied to the subjects.

In this study, the ability of the NLR to indicate anterior uveitis in BD patients was much stronger than other markers. In one of our previous publications we saw that the NLR changed significantly in the BD and BD + AU groups. The median NLR values of patients in the BD group were higher than the median values in healthy volunteers, which is consistent with the literature. ${ }^{14}$ Similarly, the median NLR values in the BD + AU group were higher than in the normal healthy volunteers. Our previous work on NLR did not have this result.

We used ROC curves to compare the ability of the MPV, PLR, and NLR to indicate anterior uveitis. When the AUC is evaluated, it is seen that the NLR is better as an indicator. On the other hand, the ability of the PLR to demonstrate anterior uveitis was found to be better than that of the MPV, but neither was statistically significant.

\section{Conclusion}

No specific tests can directly diagnose BD or its complications. Several studies have focused on the parameters which could solve this problem. The PLR and MPV have been compared, as well as the NLR, which is known to predict anterior uveitis; the NLR was found to be a better marker for detection of anterior uveitis in patients with BD than the MPV or PLR. For clinicians, one of the consequences of these results is that when they witness changes in these parameters, they should consider that one of the causes of the developing inflammation could be an anterior uveitis attack.

\section{Disclosure}

The authors report no conflicts of interest in this work.

\section{References}

1. Bolster MB. MKSAP 15 Medical Knowledge Self-assessment Program: Rheumatology. Philadelphia, PA: American College of Physicians; 2009.

2. Behçet H, Matteson EL. On relapsing, aphthous ulcers of the mouth, eye and genitalia caused by a virus. 1937. Clin Exp Rheumatol. 2010; 28(4 Suppl 60):S2-S5.
3. [No authors listed]. Criteria for diagnosis of Behçet's disease. International Study Group for Behçet's Disease. Lancet. 1990;335(8697):1078-1080.

4. Jabs DA, Nussenblatt RB, Rosenbaum JT; Standardization of Uveitis Nomenclature (SUN) Working Group. Standardization of uveitis nomenclature for reporting clinical data. Results of the First International Workshop. Am J Ophthalmol. 2005;140(3):509-516.

5. Avci A, Avci D. Serum prolactin levels in Behçet's disease. Is there a relationship between Behçet's disease and prolactin as in other autoimmune diseases? Acta Dermatovenerol Croat. 2013;21(1):52-53.

6. Türkcü FM, Yüksel H, Sahin A, et al. Mean platelet volume in pseudoexfoliation syndrome and glaucoma. Eur J Ophthalmol. 2014;24(1): $71-75$.

7. Ricart JM, Espana F, Navarro S, Todolí J, Miguel De la Fuente J, Vayá A. Mean platelet volume does not seem to relate to thrombosis or posterior uveitis in Behçet's disease. Clin Hemorheol Microcirc. 2013;54(1): 51-57.

8. Smith RA, Bosonnet L, Ghaneh P, et al. The platelet lymphocyte ratio improves the predictive value of serum CA19-9 levels in determining patient selection for staging laparoscopy in suspected periampullary cancer. Surgery. 2008;143(5):658-666.

9. Asher V, Lee J, Innamaa A, Bali A. Preoperative platelet lymphocyte ratio as an independent prognostic marker in ovarian cancer. Clin Transl Oncol. 2011;13(7):499-503.

10. Sunbul M, Gerin F, Durmus E, et al. Neutrophil to lymphocyte and platelet to lymphocyte ratio in patients with dipper versus non-dipper hypertension. Clin Exp Hypertens. 2014;36(4):217-221.

11. Gary T, Pichler M, Belaj K, et al. Platelet-to-lymphocyte ratio: a novel marker for critical limb ischemia in peripheral arterial occlusive disease patients. PLoS One. 2013;8(7):e67688.

12. Oylumlu M, Doğan A, Oylumlu M, et al. Relationship between platelet to lymphocyte ratio and coronary slow flow. Anatol J Cardiol. 2015;15(5): 391-395.

13. Gürler A, İdil A, Boyvat A, Çalıșkan D. [The evaluation of age at onset of the first symptom according to sex in the 2175 Behçet's Patients followed up between 1976 and 1997 at Ankara University Multidisciplinary Behçet's Disease Center]. Turkiye Klinikleri Journal of Dermatology. 2000;10:83-86. Turkish.

14. Rifaioglu EN, Bülbül Sen B, Ekiz Ö, Cigdem Dogramaci A. Neutrophil to lymphocyte ratio in Behçet's disease as a marker of disease activity. Acta Dermatovenerol Alp Pannonica Adriat. 2014;23(4):65-67.

15. Avci A, Avci D, Cinar SL, et al. Effect of anterior uveitis in neutrophil to lymphocyte ratio. International Eye Science. 2016;16(4).

16. Abu-Ameerh MA, Mohammed SF, Mohammad MT, Ababneh OH, Al-Bdour MD. Ocular manifestations of Behçet's disease in Jordanian patients. Saudi J Ophthalmol. 2013;27(4):247-251.

17. Kobayashi M, Ito M, Nakagawa A, et al. Neutrophil and endothelial cell activation in the vasa vasorum in vasculo-Behcet's disease. Histopathology. 2000;36(4):362-371.

18. Eksioglu-Demiralp E, Direskeneli H, Kibaroglu A, Yavuz S, Ergun T, Akoglu T. Neutrophil activation in Behcet's disease. Clin Exp Rheumatol. 2001;19(5 Suppl 24):S19-S24.

19. Zierhut M, Mizuki N, Ohno S, et al. Immunology and functional genomics of Behçet's disease. Cell Mol Life Sci. 2003;60(9):1903-1922.

20. Becatti M, Emmi G, Silvestri E, et al. Neutrophil activation promotes fibrinogen oxidation and thrombus formation in Behçet disease. Circulation. 2016;133(3):302-311.

21. Pay S, Şimşek I, Erdem H, Dinç A. Immunopathogenesis of Behcet's disease with special emphasize on the possible role of antigen presenting cells. Rheumatol Int. 2007;27(5):417-424.

22. Kawakami T, Ohashi S, Kawa Y, et al. Elevated serum granulocyte colony-stimulating factor levels in patients with active phase of sweet syndrome and patients with active Behçet disease: implication in neutrophil apoptosis dysfunction. Arch Dermatol. 2004;140(5):570-574.

23. Ekiz O, Balta I, Sen BB, Rifaioglu EN, Ergin C, Balta S, Demirkol S. Mean platelet volume in recurrent aphthous stomatitis and Behçet disease. Angiology. 2014;65(2):161-165. 
24. Lindeman S, Kramer B, Seizer P, Gawaz M. Platelets, inflammation and atherosclerosis. J Thromb Haemost. 2007;5(Suppl 1):203-211.

25. Gürsoy OM, Karakoyun S, Kalçik M, et al. Usefulness of novel hematologic inflammatory parameters to predict prosthetic mitral valve thrombosis. Am J Cardiol. 2014;113(5):860-864.

26. Threatte GA. Usefulness of the mean platelet volume. Clin Lab Med. 1993;13(4):937-950.

27. Acikgoz N, Karincaoglu Y, Ermis N, et al. Increased mean platelet volume in Behçet's disease with thrombotic tendency. Tohoku J Exp Med. 2010;221(2):119-123.

28. Kisacik B, Tufan A, Kalyoncu U, et al. Mean platelet volume (MPV) as an inflammatory marker in ankylosing spondylitis and rheumatoid arthritis. Joint Bone Spine. 2008;75(3):291-294.

29. Yuksel O, Helvaci K, Basar O, et al. An overlooked indicator of disease activity in ulcerative colitis: mean platelet volume. Platelets. 2009;20(4):277-281.

30. Uzkeser H, Haliloglu S, Cayir Y, et al. Is mean platelet volume a new activity criterion in Behçet's disease? Blood Coagul Fibrinolysis. 2015;26(7):836-839.

31. Alan S, Tuna S, Türkoğlu EB. The relation of neutrophil-to-lymphocyte ratio, platelet-to-lymphocyte ratio, and mean platelet volume with the presence and severity of Behçet's syndrome. Kaohsiung J Med Sci. 2015; 31(12):626-631.

32. Azab B, Shah N, Akerman M, McGinn JT Jr. Value of platelet/ lymphocyte ratio as a predictor of all-cause mortality after non-STelevation myocardial infarction. J Thromb Thrombolysis. 2012;34(3): 326-334.

33. Koide N, Saito H, Suzuki A, et al. Clinicopathologic features and histochemical analyses of proliferative activity and angiogenesis in small cell carcinoma of the esophagus. J Gastroenterol. 2007;42(12):932-938.

34. Brenner B, Tang LH, Shia J, Klimstra DS, Kelsen DP. Small cell carcinomas of the gastrointestinal tract: clinicopathological features and treatment approach. Semin Oncol. 2007;34(1):43-50.
35. Tamhane UU, Aneja S, Montgomery D, Rogers EK, Eagle KA, Gurm HS. Association between admission neutrophil to lymphocyte ratio and outcomes in patients with acute coronary syndrome. Am J Cardiol. 2008;102(6):653-657.

36. Zahorec R. Ratio of neutrophil to lymphocyte counts - rapid and simple parameter of systemic inflammation and stress in critically ill. Bratisl Lek Listy. 2001;102(1):5-14.

37. Knaus WA, Draper EA, Wagner DP, Zimmerman JE. APACHE II: a severity of disease classification system. Crit Care Med. 1985;13(10): 818-829.

38. Vincent JL, Moreno R, Takala J, et al. The SOFA (Sepsis-related Organ Failure Assessment) score to describe organ dysfunction/failure. On behalf of the Working Group on Sepsis-Related Problems of the European Society of Intensive Care Medicine. Intensive Care Med. 1996;22(7):707-710

39. Nakanishi N, Sato M, Shirai K, Suzuki K, Tatara K. White blood cell count as a risk factor for hypertension; a study of Japanese male office workers. J Hypertens. 2002;20(5):851-857.

40. Marsland AL, McCaffery JM, Muldoon MF, Manuck SB. Systemic inflammation and the metabolic syndrome among middle-aged community volunteers. Metabolism. 2010;59(12):1801-1808.

41. Cananzi FC, Dalgleish A, Mudan S. Surgical management of intraabdominal metastases from melanoma: role of the neutrophil to lymphocyte ratio as a potential prognostic factor. World J Surg. 2014;38(6): $1542-1550$

42. Balkarli A, Kucuk A, Babur H, Erbasan F. Neutrophil/lymphocyte ratio and mean platelet volume in Behçet's disease. Eur Rev Med Pharmacol Sci. 2016;20(14):3045-3050.

43. Yuksel M, Yildiz A, Oylumlu M, et al. Novel markers of endothelial dysfunction and inflammation in Behçet's disease patients with ocular involvement: epicardial fat thickness, carotid intima media thickness, serum ADMA level, and neutrophil-to-lymphocyte ratio. Clin Rheumatol. 2016;35(3):701-708.
Therapeutics and Clinical Risk Management

\section{Publish your work in this journal}

Therapeutics and Clinical Risk Management is an international, peerreviewed journal of clinical therapeutics and risk management, focusing on concise rapid reporting of clinical studies in all therapeutic areas outcomes, safety, and programs for the effective, safe, and sustained use of medicines. This journal is indexed on PubMed Central, CAS,

\section{Dovepress}

EMBase, Scopus and the Elsevier Bibliographic databases. The manuscript management system is completely online and includes a very quick and fair peer-review system, which is all easy to use. Visit http://www.dovepress.com/testimonials.php to read real quotes from published authors. 